\title{
SWITHSONIAN LIBRARIES
}





r. W. UEVUE A G. I. RAYNOLDS CO. NEW YORK AND CHICAGO.

MANUFACTURES OF

\section{Prepared Canvas for Oil Painting,}

\section{Academy Boards, Oil Sketching Paper}

Supplies for Oil and Water Color Painting. Pastel and Miniature Painting, Charcoal and Crayon Drawing, Sketching, Designing, Etching, Modeling, Etc.

OILS, VARNISHES AND MEDIUMS, FINE BRUSHES FOR OIL AND WATER-COLOR PAINTING.

Pastel Canyas axd Pastel Board. Models, Books ox Art and Studies.

F. W. Devoe \& Co.'s Prepared Oil Colors for Artists.

\section{THE \\ Cong Island Coan and Crust Company} 203 MONTAGUE STREET, BROOKLYN, N. Y.

\section{Capital, $\$ 500,000.00$ \\ Surplus, $\$ 400,000.00$.}

This surplus has accumulated from the successful administration of the business of the company since its organization in 1884 , and has not in any way been created by contributions from stockholders.

INTEREST ALLOWED ON DEPOSITS.

i

EDWARD MERRITT, President.

DAVID G. LEGGET, Vice-President. FREDERICK T. ALDRIDGE, Secretary.

Watson B. Dickerman, William M. Ingraham, Bernard Peters.

Edward D. White.

David Barnett.

Frank L. Babbott,

TRUSTEES.

John F. Halsted,

George Edward Ide.

Edward Merritt,

Frank Lyman,

David G. Legget,
Seymour L. Husted, Jr.

James W. Cromwell, Theodore F. Jackson, William C. Redfield, Seth L. Keeney. 

REBER

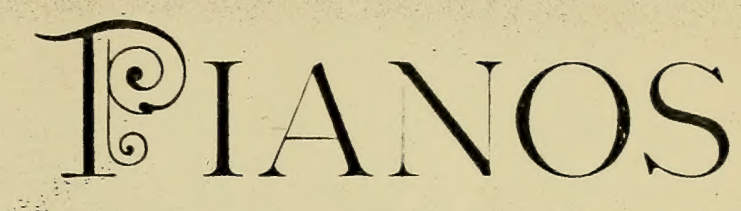

Q

The distinguishing char-

i acteristic of the Weber Piano is its Sympathetic Tone.

WAREROOMS:

Fifth Avenue, cor. Sixteenth Street,NEW YORK.

$25^{8} \& 260$ Wabash Avenue, CHICAGO. 




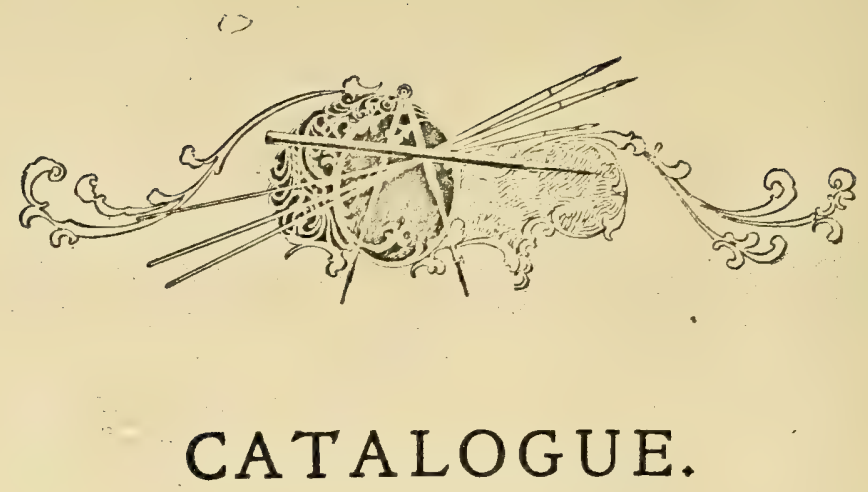

LOANED BY

Joseph C. Hoagland.

No. 1.

LANDSCAPE AND CATTLE-Approaching Storm.

Constant Troyon, - - $\quad$ - $\quad$ - $\quad$ - $\quad$ Paris

No. 2.

MARINE.

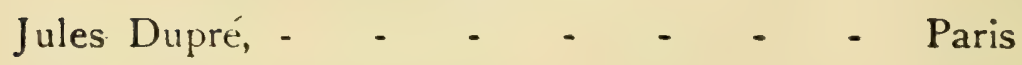

No. 3 .

COMMENTING.

A. Neuhuys, - _ _ _ _ _ - Holland

No. $f$ :

A BRITTANY GIKI.

Jules Breton, - - _ _ _ - - - Paris 


\section{No. 5 .}

THE SHEEPFOII).

Charles Jacque,

Paris

No. 6.

DR. WALKEK, Founder of the London Botanical Garden

D. Hains, - • • - _ _ - London

No. 7 .

LANDSCAPE.

Charles François Daubigny, - - _ _ Paris

No. 8 .

DOMESTIC LIFE IN HOLLAND,

A. Neuhuys, - _ _ _ _ . Holland

No. 9 .

PORTKAIT OF A GENTLEMAN.

Sir Anthony Van Dyck, - . - . London

No. Io.

PORTRAIT.

Wm. Hogarth, - - - - - London

No. II.

IDEAL PASTIMES.

A. Monticelli, - $\quad$ - $\quad$ - $\quad$ - . Paris

No. 12.

PORTRAIT OF A IAAIY.

Sir Thomas Lawrence, - _ _ _ _ London 


\section{No. 13. \\ IN THE GARDEN.}

A. Monticelli, - $\quad-\quad-\quad \ldots \quad-\quad-\quad$ Paris

No 14.

LANDSCAPE.

Geo. Barret,' - _ _ _ _ _ _ London

No. 15.

WATCHING THE COWS.

Jules Breton, - $\quad$ - $\quad$ - $\quad$ - $\quad$ - Paris

No. 16.

DUKE OF DEVONSHIRE.

Henry William Pickersgill. - _ - London

No.: 17.

IN THE FOREST.

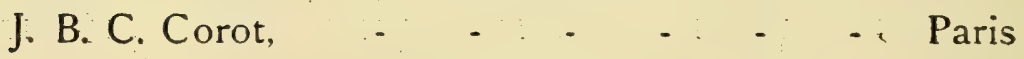

No. IS.

HOLY FAMILY.

Sir Joshua Reynolds, - _ _ _ _ London

No. 19.

THE PLAINS OF BARBIZON.

Leon Richet, - . . . _ . . Paris

No. 20.

FRENCH VILLAGE.

Theodore Rousseau, - _ _ . Paris 
No. 2 I.

LAN DSCAPE.

George Michel, _ $\quad \ldots \quad \ldots \quad$ - $\quad$ - $\quad$ - $\quad$ Paris

No. 22.

PORTRAIT OF WILLIAM SHAKESPEARE.

No. 23.

ENGLISH LANDSCAPE.

Frederick Watts, - $\quad$ - $\quad$ - $\quad$ - $\quad$ - London

Pupil of John Constable.

No. 24 .

CLASSIC LANDSCAPE.

Sir George Howland Beaumont, - - London

No. 25.

VIEIV NEAR NORIVICH.

John Birnay Crome, _ _ _ _ _ _ _ _ _ London

No. 26.

A WOODLAND SCENE.

Thomas Gainsborough, - _ _ _ London

No. 27 .

AT THE SPRING.

William Collins, . - $\quad$ - $\quad$ - $\quad$ - $\quad$ - $\quad$ London

No. 28 .

PASTORAL, LANDSCAPE.

N. Diaz, - - - $\quad$ - $\quad$ - $\quad$ - $\quad$ - Paris 
No. 29.

STREET IN LAREDO, SPAIN.

Mark Antony,

No. 30 .

NORMANDY COW.

Emil Van Marcke, _ _ _ _ . . Paris

No. 3 I.

L'INCROYABLE.

Leo Herrmann, - $\quad$ - $\quad$ - $\quad$ - $\quad$ - $\quad$ - Paris

No. 32 .

ENGLISH LANDSCAPE.

Yeend King, - $\quad$ - $\quad$ - $\quad$ - $\quad$ - $\quad$ - $\quad$ London

No. 33 .

THE, END OF THE MONTH OF MAY.

C. F. Daubigny, - _ _ _ _ _ _ - Paris

No. 34 .

FOREST OF FONTAINEBLEAU.

N. Diaz, - - - - - - - - Paris

No. 35 .

RUSSIAN WINTER SCENE.

A. W. Kowalski, - _ _ _ _ _ Paris

No. $3^{6}$.

FOREST OF FONTAINEBLEAU.

Theodore Rousseau,

- Paris 


\section{No. 37 .}

\section{SPANISH WOLF-HOUND.}

John Phillip, - - $\quad$ - $\quad$ - $\quad$ - London

No. $3^{S .}$

THE GYPSY ENCAMPMENT.

Thomas Gainsborough, - - - - _ London

No. 39.

ARAB HORSEMAN.

A. Schreyer, - - - - - - - Paris

No. 40.

IDEAL HEAD.

J. J. Henner, - - - - - $\quad$ - - - Paris

No. 41 .

SHEEP.

Charles Jacque, - - $\quad-\quad-\quad-\quad-\quad-$ Paris

No. $4^{2}$.

AN INTERVIEW WITH HIS HONOR.

Erskin Nicol,

London

No. 43 .

FRAU VAT'TLES' HOME.

H. W. Ranger, - - - - - New York

No. 44 .

CATTLE IN LANDSCAPE.

Ogden Wood, - - - - - - New York 


\section{No. 45 .}

LANUSCAPE.

George Michel, - -

THE INCORRIGIBLE.

John Burr, - _ _ _ _ _ _ London

No. 47.

THE U. S. SHIP CONSTITUTION.

James G. Tyler, _ _ _ _ _ _ - New York

No. 48 .

ST. PAUL'S FRON HIGHGATE.

J. O'Connor, - _ _ _ _ _ _ London

No. 49.

PORTRAIT OF A NOBLEMAN.

Sir Godfrey Kneller, - _ _ _ _ _ L Londón

No. $5^{\circ}$.

ROMAN EMPEROR.

No. 5 I.

ROMAN EMPEROR.

No. 52.

- BURNING THE WEEIS.

Henry Lerolle, - 


\section{No. 53 .}

KING ALFRED IN THE NETHERLANDER'S HUT.

Gerbrand Van Den Eeckhout, - - Holland

Pupil of Rembrandt.

$$
\text { No. } 54 \text {. }
$$

EVENING LANISCAPE.

E. J. Niemann, - $\quad-\quad-\quad-\quad-\quad$ Munich

\section{No. 55 .}

THE FAITHFUL GUARDIAN.

Marei Ten Kate, - $\quad$ - $\quad$ - $\quad$ - $\quad$ - $\quad$ - Paris

$$
\begin{array}{ll} 
& \text { No. } 56 . \\
\text { RETURNING FROM THE FIELDS. }
\end{array}
$$

A. Mollinger, - - - - $\quad--_{-} \quad-$ Paris

No. 57.

VIEW OF LAKE NEMI,

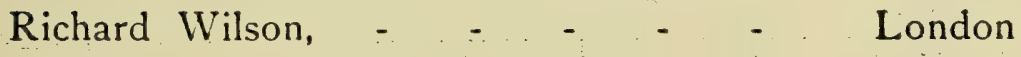

$$
\begin{aligned}
& \text { No. } 5^{8} . \\
& \text { COAST OF MAINE. }
\end{aligned}
$$

F. K. M. Rehn, - - - - - - New York

$$
\text { No. } 59 .
$$

THE FIRST STAGE COACH FROM WINCHESTER TO PORTSMOUTH, ENGLAND.

J. F. Herring, Sr., - - _ _ _ - London

No. 60 .

RECRUITING.

Herman Ten Kate, - _ _ _ _ - Paris 


\section{No. 61 . \\ STREET IN SEVILLE.}

Mark Antony,

\section{No. 62. \\ DRESSING THE BABY.}

A. Neuhuys, - - _ _ - _ Holland

No. 63.

WINTER EVENING.

Jose Heydendahl, - _ _ _ _ _ Holland

No. 64 .

THE PHILOSOPHER.

Josef Israels, - _ _ _ _ _ _ _ Holland

LOANED BY

A. Augustus Healy.

No. 65 .

A DUTCH FISHERIOMAN.

Franz Hals, - _ _ _ . _ _ Holland

No. 66 .

CLEARING AFTEK RAIN.

Jacob Maris, - $\quad$ - $\quad$ - $\quad$ - $\quad$ - Holland

No. $6 \%$

DRAWING CANAL BOATS AT TWILIGHT.

Jacob Maris, - $\quad$ - $\quad$ - $\quad$ - $\quad$ Holland 
No. 68 .

UNDER THE WILI.OWS.

Wm. Maris. - _ _ _ _ _ _ Holland

No. 69 .

AUTUMN LANISSCAPE.

Theodore Rousseau,

Paris

No. 70.

LANDSCAPE IN HOLLAND.

W. Roelofs, - - _ _ _ - $\quad$ - Holland

No. 71 .

RETURNING FLOCK IN WINTER.

F. P. Ter Meulen, - _ $\quad$ - $\quad$ - $\quad$ - $\quad$ - Holland

No. 72.

SISTER AND BROTHER.

J. S. H. Kever, - . - - _ _ - Holland

No. 73 .

VIEIV OF DORDRECHT (Water Color).

Jacob Maris, - $\quad$ - $\quad$ - $\quad$ - $\quad$ - Holland

No. 74 .

AN ELDERLY WOMAN (Water Color).

D. A. C. Artz, - - - _ _ Holland

No. 75 .

MEADOWS IN SPRING (Water Color).

Geo. Poggerbeck, - - - - - - $\quad$ - Holland 


\section{No. 76 .}

CANAL IN HOLLAND (Water Color).

F. J. Du Chattel, - - _ _ - - Holland

No. 77.

FEEDING BABY (Water Color).

J. S. H. Kever, - - - - - Holland

\section{LOANED BY \\ Catholina Lambert.}

No. 7 .

GIRL WITH PARROT.

Caroline Reed, - _ _ _ _ _ _ $\quad$ - London London.

The only lady artist ever elected to membership in the Royal Academy,

No. 79 .

THE SISTERS.

Sir Thomas Lawrence, - $\quad$ - _ _ London

No. So.

THE PRICE FAMILY.

William Hogarth, - $\quad$ - $\quad$ - $\quad$ - $\quad$ - London

No. SI.

PORTRAIT OF A FRENCH LADY.

No. $S_{2}$.

AUTUMN 1.ANDSCAPE.

A. Monticelli, - _ _ _ _ _ Paris 
Nu. $s_{3}$.

A. Monticelli.

RUINS OF AN OLD CASTLE.

No. 84 .

THE VESTAI, VIRGINS

A. Monticelli, - - _ _ _ _ _ - Paris

No. 85 .

THE VESTAL, VIRGINS.

A. Monticelli, - _ _ _ _ _ _ _ _ $\quad$ - Paris

No. 86.

A GARDEN SCENE.

A. Monticelli, - $\quad$ - $\quad$ - $\quad$ - Paris

No. 87 .

CUPID'S OFFERING.

A. Monticelli, - _ - _ _ _ Paris

No. 88.

LANDSCAPE.

A. Monticelli, - $\quad$ - $\quad$ - $\quad$ - $\quad$ - $\quad$ - Paris

No. 89 .

FIGURES IN LANDSCAPE.

A. Monticelli, - $\quad$ - $\quad$ - $\quad$ - $\quad$ - Paris

No. 90 .

THE CAVALIER.

A. Monticelli, - $\quad$ - $\quad$ - $\quad$ - $\quad$ - Paris 
No. 9 I.

HER LADYSHIP WAITING.

A. Monticelli,

No. 92.

THE JOLLY COOKS.

A. Monticelli,

$$
\begin{aligned}
& \text { No. } 93 . \\
& \text { FEEDING THE CHICKENS. }
\end{aligned}
$$

A. Monticelli, _ - _ _ _ _ _ _ _ Paris

No. 94 .

A LADY'S PORTRAIT.

A. Monticelli,

LOANED BY

Latham A. Fish.

No. 95 .

GANSETOORT SQUARE, NEW YORK.

Henry W. Ranger, - - _ _ - New York

No. 96 .

MARKET DAY IN POLAND.

A. Kowalski, - _ _ $\quad$ - $\quad$ - $\quad$ - $\quad$ - Russia

No. 97 .

AN APPROACHING STORM.

James M. Hart, - - _ _ - New York 

A. Cary Smith.

No. 98 .

"THE AGNES."

No. 99.

BAVARIAN PEASINT GIRL.

E. Raw, - - - - $\quad$ - $\quad$ - $\quad$ - $\quad$ - $\quad$ - Munich

No. 100.

MORNING TOILET.

Rudolph Epp, - $\quad$ - $\quad$ - $\quad$ - $\quad$ - $\quad$ - Munich

No. ror.

ON THE RIYER AVON.

R. W. Van Boskerk, - - - - New York

No. 102.

LIMBER UP.

T. De Thulstrup, - - - - - - New York

No. í3.

THE NEI SCHOLAR.

B. Vautier, - - - $\quad$ - $\quad$ - $\quad$ - Dusseldorf

No. 104.

OLIVER TWIST.

L. Ruel, - - - - - - - - Paris

No. 105.

LATHAM AVERY.

Waldo, - - - - - - - United States 


\section{No. 106.}

DEBORAH AVERY,

Waldo, - - $\quad$ - $\quad$ - $\quad$ - United States

No. 107.

NARRAGANSETT COAST.

F. A. Silva, - - - - - - New York

No. 108.

OLD OAKS.

H. P. Smith, - - - - - - New York

No. 109 .

WINTER.

F. H. Kammerer, - $\quad$ - $\quad$ - $\quad$ - $\quad$ - $\quad$ Paris

No. IIo.

WALLACHIAN TEAMSTERS.

A. Schreyer, - $\quad$ - $\quad$ - $\quad$ - $\quad$ - $\quad$ Paris

No. III.

LANDSCAPE.

W. T. Richards, - $\quad$ - $\quad$ - $\quad$ - Philadelphia

No. 112.

THE AMBLSCADE.

IV. Velton, - $\quad$ - $\quad$ - $\quad$ - $\quad$ - $\quad$ - $\quad$ - Munich

No 113.

LATF IFTERNOON.

1. Pokitinow, - $\quad$ - $\quad$ - $\quad$ - $\quad$ - Paris 
No. IIt.

THE CARDINAL.

J. G. Vibert, - - - - - - - - Paris

No. 115 .

CROSSING THE FIELDS.

Jules Breton, - $\quad$ - $\quad$ - $\quad$ - $\quad$ - $\quad$ - $\quad$ - Paris

No. 116.

A SPANISH FAIR.

José Jiminez Aranda, - $\quad$ - _ _ _ $\quad$ - Madrid

No. 117.

WINTER IN RUSSIA.

Szykier Siekierz, - - - - - - -

No. II 8 .

INTERRUPTED.

W. Geetz, - $\quad-\quad-\quad-\quad-\quad-\quad$ Munich

No. 1 I 9.

GARDEN IN THE LAGOON, VENICE.

M. Rico, - $\quad$ - $\quad$ - $\quad$ - $\quad$ - $\quad$ - $\quad$ -

No. 120.

A LADY'S PORTRAIT.

C. Jacquet, - - - - - - - - Paris

No. $12 \mathrm{r}$.

A GOOD STUDY.

E. Zimmermann, 
No, 122.

THE SIVING.

Geo. H. Boughton,

London

No. 123.

THE FIRST SORROW.

Meyer von Bremen

Bremen

No. 124.

THE CARDINAL'S RETURI.

J. G. Vibert, - - _ _ _ - - _ Paris

No. 125 .

WAITING.

Firman Gerard, - - - - _ _ Paris

No. 126.

GIRL'S HEAD.

G. Jacquet, - $\quad-\quad-\quad-\quad-\quad-$ Paris

No. 127.

INN ON THE AVON.

G. H. McCord, - - - - - New York

No. $12 S$.

ON THE HACKENSACK.

W. S. Macy, - - - - - - New York

No. 129 .

LANDSCAPE.

A. W. Casalear, - $\quad$ - $\quad$ - $\quad$ - New York 


\section{No. 130.}

THE FISHERMIN'S DAUGHTER.

Leon Moran, - _ _ _ _ _ _ _ New York

No. 13 I.

A CAYALIER.
A. Gues, -

No. 132.

SULLIYAN COLNTY.

H. P. Smith, - - - _ _ _ - New York

No. 133.

HOLLANI) FISHERIVIMA.

F. Miller, - - _ _ _ _ _ - New York

No. 134 .

NEW ENGLAND SCENERY.

H. P. Smith, - - - - - - - - New York

No. 135 .

BATTLE OF WINCHESTER.

T. De Thulstrup, - - _ _ - - New York

No. 136.

HORSE ARTIILERY.

T. De Thulstrup, - - - - - - New York

No. 137.

HORNETS' NEST, SHILOH.

T. De Thulstrup, - - - - - _ - New York 


\section{No. 138.}

THE RLOONY ANGFI, SPOTTSYLVANIA.

T. De Thulstrup, - $\quad$ - $\quad$ - $\quad$ - _ New York

No, 139.

LOGAN AT KENNESAII.

T. De Thulstrup, - _ _ _ - New York

No. 140.

ALTOONA.

T. De Thulstrup, - _ _ _ _ - New York

No. $1+1$.

SHERMAN AT SIEGE OF ATLANTA.

T. De Thulstrup, - _ _ _ _ - New York

No. 142.

SIEGE OF VICKSBLRG.

T. De Thulstrup, - _ _ _ _ _ New York

No. $1+3$.

DUNKER CHURCH, ANTIETAM.

T. De Thulstrup, - _ _ _ _ - New Jork

No. 144 .

SHERIDAN AT WINCHESTER.

T. De Thulstrup, - _ _ _ _ New York

No. $1+5$.

HANCOCK IT GETTSBLRG.

T. De Thulstrup, _ _ _ _ _ _ _ New lork 
No. 146 .

GRANT AT LOOKOUT MOUNTAIN.

T. De Thulstrup, - _ _ _ _ _ New York

No. $1+7$.

LAYING BRIDGE AT FREDERICKSBURG.

T. De Thulstrup, - - _ _ _ _ - New York

LOANED BY

John M. Amory.

No. ${ }_{4} 4$.

PORTRAIT.

Hyacinthe Rigaud, - _ _ _ _ _ _ - France

LOANED BY

Alexander Barrie.

No. 149.

A CORNER IN THE VILLAGE VEENENDAAL.

P. J. C. Gabriel, - - - - - Holland

No. 150.

GEORGIA SUNSET.

George Inness, - _ _ _ _ _ - New York

No. 151.

NEAR CROFTS, N. Y.

A. H. Wyant, - - - - - New York 


\section{LOANED BY \\ Mrs. Edwin Beers.}

No. 152.

A MAGDALENE.

LOANED BY

George C. Barclay.

No. 153.

AFTER THE RAIN.

C. F. Daubigny, - - - - - - - - Paris

No. 154 .

HAMPSTEAD HEATH.

John Constable, _ $\quad$ - $\quad$ - $\quad$ - $\quad$ - $\quad$ London

No. 155 .

THE GLEANERS.

Leon L'hermitte, - _ _ _ _ _ _ Paris

No. 156.

VENICE.

Martin Rico, - $\quad$ - $\quad$ - $\quad$ - $\quad$ - $\quad$ - _ Paris

No. 157 .

CATTLE IN PASTERE.

Constant Troyon, - $\quad$ - $\quad$ - $\quad$ - $\quad$ - $\quad$ - Paris 
No. $1 ; 8$.

RETURN TO THE FOLD

Anton Maure, _ $\quad$ - $\quad$ - $\quad$ - $\quad$ - $\quad$ - Paris

No. 159 .

FOREST OF FONTAINEBLEAU.

N. Diaz, - - _ _ _ _ _ - _ Paris

LOANED BY

William H. Cummings.

No. 160.

AFTER THE HUNT.

Gerard Dow. - $\quad$ - $\quad$ - $\quad$ - $\quad$ - Holland

No. 16 .

FLOIVERS.

John Van Huysum, _ $\quad$ - $\quad$ - $\quad$ - $\quad$ Holland

No. 162.

DOGE,

Richard Creifelds, - _ _ _ _ _ - New York

No. $16_{3}$.

GOOD FRIENDS.

G. IV. Brenneman, - - - - New York 


\title{
LOANED BY
}

\section{Albert Kingsland Kenyon.}

\author{
No. 164 . \\ MARY MAGDALENE.
}

Nicholas Poussin, - $\quad$ - $\quad$ - $\quad$ - $\quad$ - $\quad$ - Italy

LOANED BY

Theo. A. Cole.

No. 165 .

PROMETHEUS BOUND.

Thomas Cole, - _ _ _ _ _ New York

LOANED BY

John M. Conklin.

No. 166.

LANDSCAPE AND CATTLE.

Peeter Bout, -

No. $16 \%$.

HUNTING SCENE.

Jan Baptiste IVeenix, - $\quad$ - _ _ $\quad$ - Holland

No. 168.

THE VIII.AGE SCHOOL.

F. de Brackelecr, - _ _ _ _ _ . Holland 


\section{LOANED BY \\ John Emmans.}

No. 169.

VENICE.

Ruben Santoro, - $\quad \ldots \quad \ldots \quad \ldots \quad$ - $\quad$ - Paris

No. 170.

OLD RUINS.

J. C. Cazin, - - - - - - - Paris

No. 171.

THE FARM.

Jules Dupré, - - $\quad$ - $\quad$ - $\quad$ - - Paris

No. 172.

HARVESTING.

R. B. Goddard, - _ _ _ _ _ $\quad$ London

No. 173.

SARA BERNHARDT AS MRS. CLARKSON.

Georges Clairin, - - $\quad$ - $\quad$ - $\quad$ - $\quad$ Paris

No. 174 .

AUSTRIAN PRISONERS FORCED TO WORK.

Horace Vernet, - $\quad$ - $\quad$ - $\quad$ - $\quad$ - $\quad$ - Paris

No. 175 .

THE PET.

Emile Pinchart, - _ _ _ _ _ _ - Paris 


\section{LOANED BY \\ Stephen H. Mills.}

No. 176 .

PORTRAIT OF MARTHA WASHINGTON

Gilbert Stuart, - _ _ _ - United States

LOANED BY

Mrs. Phebe A. Hearst.

No. 177 .

LITTLE PRINCESS.

Franz Lenbach, - $\quad$ - $\quad \ldots \quad$ - $\quad$ - Munich

No. 178 .

SUNSHINE

Carl Marr, - _ _ _ _ _ _ San Francisco

No. 179.

COUNTRY ROAD.

Henri Harpignies, - $\quad$ - $\quad$ - $\quad$ - $\quad$ - $\quad$ - Paris

No. ISo.

LANDSCAPE.

Wm. Keith, - $\quad$ - $\quad$ - $\quad$ - $\quad$ - San Francisco

LOANED BY

Mrs. Walter T. Hatch.

No. IS I.

PORTRAIT OF THE LATE WALTER TILDEN HATCH. Rebeca Taylor Porter, - New Haien, Conn. 


\section{LOANED HY}

\section{A. B. Lounsbery.}

No. 182.

SHEEP, Etc.

Eugene Verboeckhoven, _ _ . .

No. 183.

SCENE IN FAIRFIELD COLNTY, CONN.

W. M. Brown, - _ _ _ _ - New York

\section{LOANED BY}

Charles C. Markham.

No. 184 .

GENERAL JOHN C. FREMONT.

Charles Elliott, _ _ _ _ - United States

No. 185 .

GENERAL ANDREW JACKSON.

Charles IVilson Peale, - _ _ _ United States

No. 186.

EDWIN FORREST.

Nephew of B. West, - - - - United States

No. I 87.

PORTRAIT OF A GENTIEMAN.

Henry Inman, _ _ _ _ _ _ United States 


\section{No. 188}

PORTRAIT OF A IAIDY.

George A. Baker, - - _ - - United States

No. 189 .

PORTRAIT OF A LADY.

Oliver Stone, - _ _ _ _ _ _ _ United States

No. 190.

PORTRAIT OF A GENTLEMAX.

Oliver Stone, - $\quad$ - $\quad$ _ $\quad$ _ $\quad$ - $\quad$ United States

No. Igr.

PORTRAIT OF A GENTLEMAN.

Charles Elliott, - _ _ _ _ _ United States

No. 192.

PORTRAIT OF A GENTLEMAN.

Charles Elliott, _ _ _ _ _ - United States

LOANED BY

Joseph Jefferson.

No. 193.

LANDSCAPE.

Joseph Jefferson, - - - Buzzards Bay, Mass.

No. 194.

I.ANDSCAPF.

Joseph Jefferson, - - - Buzzards Bay: Mass 


\section{LOANED BY}

Wm. W. Rossiter.

No. 195.

PORTRAIT OF L. 'T. ROSSITER.

Daniel Huntington, - - - - - - New York

LOANED BY

Mrs. Julia G. Sutton.

No. 196.

VIEW OF THE HUDSON.

T. Doughty, - - - - - - - New York

No. 197

THE CONVERSATION.

E. H. May, - - - - - - - New York

- No. 198 .

LANDSCAPE.

D. Huntington, - - - - - - New York

LOANED BY

David McCosker.

No. 199.

PORTRAIT OF SIR JOSHUA REYNOLDS.

Rembrant Peale, - _ _ _ _ $\quad$ United States

No. 200.

THE LATEST NEWS.

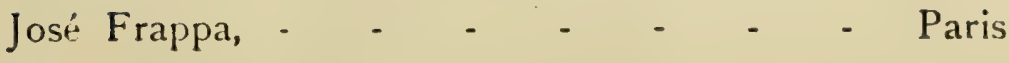


No. $20 \mathrm{I}$.

SHEEP AND LAMB.

Louis Robbe, - _ _ _ _ _ _ _ Brussels

No 202.

TROPICAL LANDSCAPE.

L. R. Mignot, - - - - - - Paris

No. 203.

EVENING PARTY, EIGHTEENTH CENTURY (Salon).

H. E. Reyntjens, - _ _ _ . - Dusseldorf

No. 204 .

THE POPLARS.

Leon G. Pelouse, - - _ _ _ - _ Paris

No. 205 .

THE EVENING STAR.

M. F. Jacomin, - _ - _ _ _ Paris

No. 206.

OUT FOR A BRUSH.

William Barrand, - _ _ _ _ _ - Paris

No. 207 .

IN THE GLOAMING.

George Inness, _ _ _ _ _ _ New York

No. 208 .

GRANDPA'S BIRTHDAY.

A. Francois, - _ _ - _ _ _ - _ - Paris

No. 209.

LANDSCAPE AND CATTLE。

Francois Lamoriniere, - _ _ _ - Paris 


\section{LOAXED BY}

A. Van Derwerken.

No. 210.

A BITTER COL,D MORNING.

C. C. Markhaim, - - _ _ - New York

\section{LOANED BY}

\section{George Mayr.}

No. 2 II.

LANDSCAPE.

Jacob Ruysdael, - $\quad$ - $\quad$ - $\quad$ - Holland

LOANED BY

Mrs. St. Clair McKelway.

No. 2 I .

PORTRAIT OF ST. CLAIR MCKELIVAY.

M. O. Newton, _ _ _ _ . . New York

LOANED BY

Hosmer B. Parsons.

No. 213.

LANDSCAPE.

William Keith, - _ _ _ San Francisco

No. $2 I_{4}$.

LANDSCAPE.

William Keith, - $\quad$ - $\quad$ - - $\quad$ San Francisco 
No. 215 .

LANDSCAPE.

William Keith - $\quad$ - _ . San Francisco

No. 216.

LANDSCAPE.

William Keith _ $\quad \ldots \quad \ldots \quad$ - San Francisco

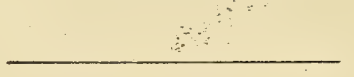

LOANED BY

F. B. Pratt.

No. 217.

SUNSET.

A. H. Wyant - - _ _ _ - New York

No. 218 .

SUNSFT.

George Inness _ _ _ _ _ _ - New York

LOANED BY

W. N. Peak.

No. 219.

THE ZENANA OF THE SLLTAN.

Ferdinand Victor-Leon Roybet - - - - Paris

$$
\text { No. } 220 .
$$

THE GLOWING SUI.

George Inness

- New York 


\section{LOANED BY}

\section{Dr. J. E. Richardson.}

No. $22 \mathrm{I}$.

INTERRUPTED COURTSHIP.

Carl Sohn - $\quad$ - $\quad$ - $\quad$ - $\quad$ - $\quad$ - Dusseldorf

No. 222.

THE FLOWER CHARITY.

Percy Moran - - - - $\quad$ - $\quad$ - $\quad$ New York

No. 223.

AUTUMN LEAVES.
A. Edelfelt
-
- New York

No. 224 .

THE MANDOLIN PLAYER.

C. Pécrus - $\quad$ - $\quad$ - $\quad$ - $\quad$ - _ _ - Paris

No. 225 .

TENNIS.

Madelaine Lemaire _ _ _ _ _ _ Paris

LOANED BY

Albert Stiener.

No. 226.

SUNSET-English Landscape.

George Inness - - - - - - New York 


\section{No. 227.}

UNDER THE OLD FIG TREE.

Jos. Lyman - _ _ _ _ _ - - New York

No. 228.

MOONLIGHT SCENE IN HOLLAND.

J. B. Jongkind - $\quad \ldots \quad \ldots \quad \ldots$ Holland

LOANED BY

Dr. S. Sherwell.

No. 229.

PORTRAIT OF AUGUSTUS GRAHAM.

First Benefactor of the Institute.

No. 230.

MUSE OF POETRY.

Copy from Carlo Dolce.

LOANED BY

William A. Towner.

No. 231.

ADORATION OF THE SHEPHERDS.

Honthorst Gerardo _ _ _ _ Holland

No. 232.

PORTRAIT OF WASHINGTON.

Gilbert Stuart

United States 
LOANED BY

Mrs. James S. Watts.

No. 233.

PASTORAL SCENE.
Philip Wouverman $\quad-\quad, \quad-\quad$ - Haerlam

PASTORAL SCENE.
Philip Wouverman - $\quad-\quad-\quad$ - Haerlam

PASTORAL SCENE.
Philip Wouverman $\quad-\quad, \quad-\quad$ - Haerlam

LOANED BY

John B. Ladd.

No. 234 .

PASTORAL IN HOLLAND.

Anton Mauve
No. 235 .
FRENCH LANDSCAPE.

F. de Mesgrigny - _ _ _ - _ _ Paris

No. $23^{6 .}$

THE HALT.

Otto de Thoren - _ _ _ _ _ _ Paris

No. 237.

FISHING BOATS OFF BELGIAN COAST.

P.J. Clays - $\quad$ - $\quad$ - $\quad$ - $\quad$ - $\quad$ - $\quad$ Brussels

No. 238.

OLD BOAT HOUSE-Venice.

B. Santoro

No. 239.

DISTRAITE-Head.

A. Claudie - $\quad$ - $\quad$ - $\quad$ - $\quad$ - $\quad$ - Paris 
No. 240 :

CLOUDY DAY.

George Inness

- New York

No. 241.

AUTUMN TWILIGHT.

A. H. Wyant - - - - - New York

No. $24^{2}$.

ST. ENFERNIA IN GUIDECCA.

M. Rico - - - - - - - - - Paris

No. 243 .

MOONLIGHT.

Richard Pauli _ $\quad$ - $\quad$ _ $\quad$ - $\quad$ - New York

No. 244.

LANDSCAPE.

Bolton Jones - $\quad-\quad \ldots \quad \ldots \quad$ New York

No. 245 .

THE DEAD FAYORITE.

L. W. Hawkins

London

$$
\begin{aligned}
& \text { No. } 2+6 . \\
& \text { SCENE IN ALGERIA. }
\end{aligned}
$$

A. Pasini - - - - - _ - - Paris

No. 247 .

SPANISH LANDSCAPE.

Sanchez-Perier - - - - _ - Paris 
No. 248 .

AT THE ANTIQUARIAN'S.

E. Grison

No. 249.

SCENE AT MANTES.

J. B. C. Corot - - - - - - Paris

No. 250.

GIRL KNITTING.

Josef Israels - -
No. $25 \mathrm{r}$.

JUDITH.

J. J. Henner: - $\quad$ - $\quad$ - $\quad$ - $\quad$ - $\quad$ - Paris

No. 252.

PASSING STORM.

Jules Dupré $\quad-\quad \ldots \quad-\quad \ldots \quad \ldots \quad \ldots \quad-\quad-\quad$ Paris

No. 253.

THE AVENUE.

Anton Mauve _ _ $\quad \ldots \quad \ldots \quad \ldots \quad \ldots$ - $\quad \ldots$ Holland

LOANED BY

John W. Mason.

No. 254.

A POOL IN THE PLAIN.

N. Diaz -

Holland 
No. 256.

COTTAGE IN THE FOREST.

Jules Dupré - $\quad$ - $\quad$ - $\quad$ - $\quad$ - $\quad$ - Paris

No. 257 .

THE FARM.

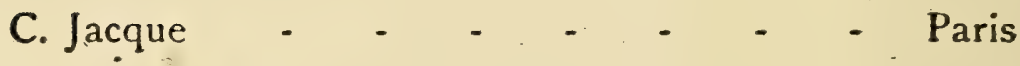

No. 258.

INNOCENCE.

A. Piot - - - - - - - - Paris

No. 259.

VENICE.

M. Rico - - - - - - - - - Paris

No. 260 .

THE : LITTLE GLEANER.

J. F. Millet - - - - - - - - Paris

No. $26 \mathrm{r}$.

PLAIN OF BARBIZON.

N. Diaz - - - - - - - - - Paris

No. 262.

SUMMER.

C. F. Daubigny - - _ - - - $\quad$ - $\quad$ - Paris

No. 263 .

FISHING BOATS.

P. J. Clays - $\quad$ - $\quad$ - $\quad$ - $\quad$ - $\quad$ - Brussels 
No. 264 .

THE BARNYARD.

E. Van Marcke _. - _ _ _ _ - Paris

No. 265.

EARLY MORNING.

J. B. C. Corot - - - - - - Paris

No. 266.

AN ARAB SHEIK.

A. G. Decamps - - - _ - - - Paris

No. 267 .

CATTLE AT POOL.

C. Jacque - $\quad-\quad+\quad-\quad-\quad \ldots \quad$ Paris

No. 268.

IN THE FOREST OF FONTAINEBLEAU.

N. Diaz - . - - - . - - - _ . Paris

No. 269 .

STRAYED FROM THE HERD.

Constant Troyon - $\quad$ - $\quad$ - $\quad$ - $\quad$ - $\quad$ Paris

No. 270.

LANDSCA PE.

Theo. Rousseau - $\quad$ - $\quad$ - $\quad$ - $\quad$ - $\quad$ - Paris

No. $27 \mathrm{I}$.

THE CONVENT IVALL.

V. Bertin 


\section{No. 272.}

\section{GYPSY ENCAMPMENT.}
A. D. Schreyer
- $\quad$ - $\quad$
$\therefore \quad$ Paris

No. 273 .

PRISCILI.A.

Thos. Hicks - - _ _ _ - - New York

No. 274 .

FRENCH VILLAGE.

J. B. C. Corot - - - - - - Paris

No. 275 .

THE COTTAGE IN THE WOODS.

George Michel _ _ _ _ _ _ _ _ Paris

No. 276.

NOONDAY REST.

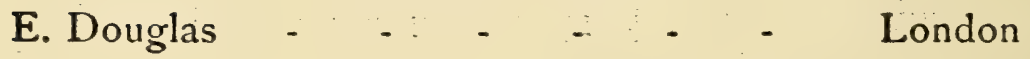

No. 277 .

A POOL IN THE FOREST.

N. Diaz -

$$
\begin{gathered}
\text { No. } 278 . \\
\text { STILL LIFE. }
\end{gathered}
$$

A. Vollon - $\quad$ - $\quad$ - $\quad$ - $\quad$ - $_{-}$- Paris

$$
\begin{aligned}
& \text { No. } 279 . \\
& \text { NEAR MONTMARTRE. }
\end{aligned}
$$

George Michel - $\quad$ - $\quad$ - $\quad$ - $\quad$ Paris 
LOANED BY

\section{De Witt Clinton Jones.}

No. 280 .

PORTR.AIT OF HON. GEORGE IV. CLINTON, A SON OF DE WITT CLINTON.

LOANED BY

Charles M. Howard.

No. $28 \mathrm{r}$.

AN INTERES'TING STORY.

F. Andriotti - - - - - - - - - Italy

No. 282 .

ON THE GRAND CANAL-VENICE.

Felix Zeim - _ _ _ _ _ _ - Paris

LOANED BY

W. D. Steel.

No. 283 .

PORTRAIT OF MRS. J. D, STEEL.

Rembrandt Peale _ _ _ _ United States

No. 284 .

PORTRAIT OF WM. STEEL.

R. Waldo

United States 
No. 285 .

PORTRAIT OF MRS. WM. STEEL.

R. Waldo - $\quad \ldots \quad \ldots \quad \ldots \quad \ldots$ United States

LOANED BY

John S. James.

No. 286.

THE DOUBTFUL BOTTLE.

W. Dendy Sadler - _ _ _ _ _

LOANED BY

Robert J. Kimball.

No. 287 .

THE MIDNIGHT SUN.

William Bradford

New York

No. 288 .

THE TABLES TURNED.

William H. Beard - - - - - New York

Leon Perrault

$$
\begin{aligned}
& \text { No. } 2 S_{9} . \\
& \text { THE FLOWER GIRL. }
\end{aligned}
$$


No. 291.

A SUMMER MORNING.

William Hart
$+$

No. 292.

IN THE BERKSHIRES.

David Johnson - _ _ _ _ _ New York

No. 293.

SPRINGTIME.

R. W. Van Boskerck _ _ _ _ - New York

No. 294.

SHEEP.

E. Verboeckhoven _ _ _ _ _

LUANED BY

Frank V. Whitney.

No. 295.

KIOSQUE D'AMURAT LE BOSPHORE, CONSTANTINOPLE.

F. Zeim - - _ _ _ _ - _ Paris

No. 296.

THE CARDINAL.

Leo Herrmann - $\quad$ - $\quad$ - $\quad$ - $\quad$ - $\quad$ - Paris

No. 297.

MIGNON.

J. G. Jacquet - - - - - - Paris 
No. 298.

FALCON HUNTING.

John Lewis Brown _ _ _ _ _ _ New York

No. 299.

A QUIET NOOK, VENICE.

M. Rico

LOANED BY

Robert B. Woodward.

No. 300.

THOUGHTS OF THE FUTURE.

. Hughes Merle - $\quad$ - $\quad$ - $\quad$ - $\quad$ - $\quad$ - Paris

No. 301 .

THE WIDOW'S COMFORT.

Prof. Wilhelm Amberg - - - - - Berlin

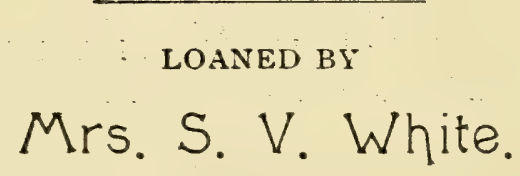

No. 302 .

A PORTRAIT.

Daisy Haven -

LOANED BY

J. E. Stillwell.

No. $3 \circ 3$.

PORTRAIT OF AARON BURR.

John Vanderlyn - - - - United States 
No. 304 .

PORTRAIT OF AARON BURR.

James Vandyck _ _ _ - . - United States

$$
\text { No. } 305 \text {. }
$$

PORTRAIT OF THEODOSIA BURR.

John Vanderlyn - $\quad$ - $\quad$ - $\quad$ United States

LOANED BY

\section{Mrs. Pierrepont.}

No. 306 .

PORTRAIT-H. E. PIERREPOON'T.

George A. Baker - - - - - New York

No. 307 .

PORTRAIT-H. B. PIERREPONT.

Henry Inman

- - - - New York

No. 308 .

PORTRAIT-GEORGE IVASHINGTON, I796.

Gilbert Stuart _ $\quad$ - $\quad-\quad \cdots \quad$ United States

LOANED BY

Carll H. De Silver.

No. $3 \circ 9$.

LANDSCAPE.

H. Harpignies - - - - - - Paris

No. 3 I0.

LANDSCAPE, KEENE VALLEY.
A. H. Wyant
- New York 
No. 311 .

MIRROR OF NATURE.

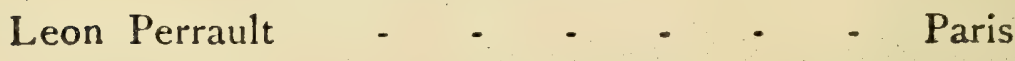

No. $3^{12 .}$

MOONRISE.

C. F. Daubigny - - - - - - - Paris

No. 3 r3.

COW'S HEAD.

Eugene Verboeckhoven _ _ _ - Brussels

LOANED BY

Mrs. J. R. Van Brunt.

M. Hobbema

\author{
No. 314. \\ GOING TO CHURCH. \\ GOING TO CHURCH.
}

M. Hobbema - - -

No. 315 .

SCENE IN NORWAY.

C. Van Everdingen - - - - : Holland

LOANED BY

William T. Evans.

No. $3^{16 .}$

RETURNING FROM PASTLRE.

George Inness

- New York 
No. 317.

NIGHT.

George Inness _ _ _ _ _ _ N New York

No. 318 .

IN THE ADIRONDACKS.

A. H. Wyant - - - - - - New York

No. 319.

AUTUMN IN THE NORTH WOODS.

A. H. IVyant $\quad-\quad \ldots \quad-\quad$ - New York

No. $3^{2 C}$.

NORMANDY TREES.

Homer D. Martin - _ _ - New York

No. 321.

AN OLD CHURCH IN NORMANDY.

Homer D. Martin

- New York

No. 322.

A NORMANDY LANDSCAPE.

Homer D. Martin - _ _ _ - New York

No. 323.

AN EAST RIVER IDYL.

H. W. Ranger - - - - - - New York

No. 324 .

A VETERAN.

H. W. Ranger - - - - - New York 
No. 325 .

A NOCTURNE.

H. W. Ranger - - - _ - New York

No. 326.

EVENTIDE.

Robert C. Minor - _ _ _ - New York

- No. 327.

MIINIGHT.

Robert C. Minor - - - - - New York

No. 328 .

THE HUNTER'S MOON.

Robert C. Minor - - - - - New York

No. 329 .

THE RETURN OF THE HAY BOATS.

Charles Melville Dewey - - - - New York

No. $33^{\circ}$.

THE HARVEST IN THE MIDLANDS.

Charles Melville Dewey - - - New York

No. $33 \mathrm{I}$.

AT PARADISE, NEIVPORT.

Samuel Colman - - - _ - New York

No. 332 .

SEA AND RAIN.

George H. Bogert - - - - - New lork 
No. 333 .

MIOONLIGH'P.

Albert P. Ryder - _ _ _ - - New York

No. 334 .

THE LITTLE MAID OF ACADIE.

Albert P. Ryder - - - - - - New York

No. 335 .

I.ENGTHENING SHADOWS.

J. Alden Weir _ - _ _ _ _ - New York

No. 336 .

DAYBREAK.

D. W. Tryon - - - - - - : New York

No. 337 .

A SPRING MORNING.

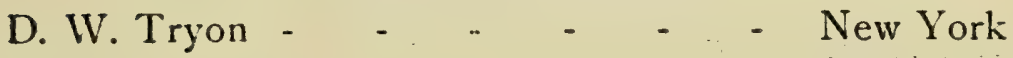

No: $33^{8}$.

THE DESERTED FARM.

J. Francis Murphy _ - _ _ _ - New York

No. 339 .

THE REFLECTION.

Benjamin R. Fitz - - _ - • N New York

No. $34^{\circ}$.

ARIADNE.

Wyatt Eaton

- - - - New York


No. 34 .

THE WATER LILIES.

Walter Shirlaw _ _ _ _ - New York

No. $34^{2}$.

THE KISS.

Walter Shirlaw - _ _ _ - New York

No. $3+3$.

AMONG THE OLD POETS.

Walter Shirlaw

\section{ANONG THE OLD POETS.}

- New York

No. 344 .

THE INDIAN HUNTER.

George de Forest Brush - _ - New York

No. $3+5$.

MADONNA AND CHILD.

Robert L. Newman, _ _ - - - . New York

No. $34^{\circ}$.

CHRIST STILLING THE TEMPEST.

Robert L. Newman _ _ _ _ New lork

No. $3+7$.

THE LETTER.

Robert L. Newman - _ _ _ _ New lork

No. $3+s$.

CHRIST IN THE WILDERNESS.

Elliott Daingerfield - _ _ - New lork 


$$
\begin{aligned}
& \text { No. } 3+9 \text {. } \\
& \text { MY LADY RHODODENIDRON. }
\end{aligned}
$$

Elliott Daingerfield - - _ - New York

No. $35^{\circ}$.

GIRL. WITH VIOLIN.

Edmund C. Tarbell - - _ - New York

No. $35 \mathrm{I}$.

BOYS PLAYING MARBLES.

Platt P. Ryder - - - _ _ _ - New York

No. $35^{2}$.

SUNDAY MORNING IN VIRGINIA.

Winslow Homer - $\quad$ - $\quad$ - New York

No. 353

MOONLIGHT.

R. A. Blakelock - $\quad-\quad-\quad-\quad \ldots$ New York

No. 354 .

THE NYMPHS.

R. A. Blakelock - - - - - New York

No. 355 .

THE MORNING VISION.

Henry Oliver Walker _ _ _ _ New York

No. $35^{6}$.

THE BOY AND THE MUSE.

Henry Oliver Walker - _ _ _ New York 
No. 357.

BRINGING HOIE THE COW.

George Fuller

New York

No. $35^{8}$.

THE RETURN TO THE FOLD.

Louis Paul Dessar

New York

No. 359 .

ONE DAY IN JUNE.

William T. Smedley

- - New York

No. 360.

ST. CECILIA.

F. S. Church - - _ _ _ - New York

No. $3^{6 r}$.

A LADY IN BLUE GOWN.

Thomas W. Dewing - $\quad-\quad-\quad$ - New York

No. 362 .

FLORÉAL.

Mowbray H. Siddons. - _ _ _ New York

No. $3^{6}$.

THE PORTRAIT.

Will H. Low

- - . - - New Iork

No. 364 .

AFTER THE FESTIVAL.

Frank D. Millet - _ _ _ - New York 
No. 365 .

CHARGING THE BATTERY.

Gilbert Gaul

LOANED BY

O. W. Faris.

No. 366 .

MOTHER AND CHII,D.

N. Diaz - - - - - - _ _ . Paris

No. 367 .

GATHERING THE SEINE.

Harry Chase - _ _ _ _ _ _ - New York

LOANED BY

Henry M Johnston.

No. 368 .

THE EMBARCATION, TIME OF LOUIS XIV.

L. G. E. Isabey - $\quad$ - $\quad$ - $\quad$ - _ - Paris

No. $3^{69}$.

SHEPHERD AND SHEEP.

Charles Jacque

No. 370 .

A CLEARING IN THE FOREST.

N. Diaz - - $\quad$ - $\quad$ - $\quad$ - $\quad$ - $\quad$ - Paris 


\section{No. $37 \mathrm{I}$.}

THE FINISHED TASK.

George Henry Harlow - _ _ _ _ London

No. 372 .

A SAILOR'S HOME.

J. C. Cazin - - - - _ - - - Paris

No. 373 .

BOURG $\cdot$ LA REINE.

J. C. Cazin - _ - _ _ _ _ - Paris

No. 374 .

MOONLIGHT IN HOLLAND.

J. B. Jongkind

Holland

No. 375 .

MOONLIGHT ON THE RIVER NEAR ANTWERP.

J. B. Jongkind _ $\quad-\quad \ldots \quad-\quad \ldots \quad \ldots \quad$ Holland

No. 376 .

AT THE POOL.

A. Watelin - _ _ _ _ _ _ _ _

No. 377 .

WINTER DESOLATION.

John J. Hasselman _ _ _ _ _ London

No. 378 .

"MORNING," THE OU'TER POR'T OF HAVRE.

E. Boudin - - _ _ _ _ _ Paris 
No. 379

THF ARTIST'S GARDEN.

E. Boudin

$$
\begin{aligned}
& \text { No. } 3^{\text {So. }} \\
& \text { ENVIRONS OF ANTIBES. }
\end{aligned}
$$

E. Boudin

$$
\begin{aligned}
& \text { No. } 3^{81} \text {. } \\
& \text { M.ARINE-DEANVILLE BEACH. }
\end{aligned}
$$

E. Boudin

$$
\begin{aligned}
& \text { No. } 3^{82 .} \\
& \text { MARINE-A BREEZY DAY. }
\end{aligned}
$$

E. Boudin

$$
\text { No. } 3^{8} 3 \text {. }
$$

GOLDEN SUNSET AT ST. VALERY.

E. Boudin - $\quad$ - $\quad$ - $\quad$ - $\quad$ - $\quad$ - $\quad$ - Paris

No. $3^{84}$.

E. Boudin

\section{- THE FORT AT ANTIBES.}

No. $3^{85}$.

MOONLIGHT AT ST. VALERY.

E. Boudin

E. Boudin

$$
\begin{aligned}
& \text { No. } 386 . \\
& \text { STREET IN VIILEFRANCHE. }
\end{aligned}
$$$$
\text { No. } 3^{87} \text {. }
$$$$
\text { MOONRISE ON THE LAKE. }
$$

E. Boudin 


\section{LOANED BY \\ Mrs. E. P. McCoy.}

No. $3^{88}$.

PORTRAIT-IVILLIAM PINKNEY, OF MARYLAND,

Ex-United States Senator, Ambassador to England, Attorney-General U. S., etc.

Rembrandt Peale

United States

No. $3^{89}$.

MRS. WILLIAM PINKNEY.

John Trumbull _ _ _ _ _ _ United States

No. 390.

CUMBERLAND D. WILLIAMS, OF BALTIMORE.

Gilbert Stuart _ _ _ _ _ _ United States

No. 39 r.

Mrs. CUMBERLAND D. WILLIAMS,

Daughter of William Pinkney.

Gilbert Stuart

United States

LOANED BI

The Estate of Wm. Hart.

No. 392 .

SCENE AT NAPANOCH, N. L. Landscape and Cattle. Wm. Hart

- New lork 
LOANED BY

\section{W. J. Smith.}

No. 393 .

A POINTER.

Charles Jacque - $\quad$ - $\quad$ - $\quad$ - $\quad$ - $\quad$ - Paris

No. 394 .

THE CIDER MILL.

W. S. Mount - _ _ _ - - New York

LOANED BY

C. M. Bul1.

No. 395 .

GIRL'S HEAD.

Jean Baptiste Greuze : - - $\quad-\quad \quad-\quad \quad \quad-\quad$ - : Paris

No. 396 .

LANDSCAPE.

M. Hobbema

- $\quad$ - $\quad$ - Holland

No. 397.

MATERNAL INSTRUCTION.

G. Netcher - -
No. $39^{8}$.

MUSICAL ASSEMBLY IN GARDEN.

Nicholas Lancret - _ _ _ _ _ _ - Paris 


\section{No. 399.}

FI,OWERS.

John Breughel _ $\quad \ldots \quad \ldots \quad \ldots \quad$ - $\quad$ Brussels

- No. 400.

FEEDING THE FLOCK.

C. Du Jardin _ _ _ _ _ _ Holland

No. 401 .

HUNTING SCENE.

Jan Weenix - _ _ _ _ _ _ Holland

No. 402 .

GABRIELLE.

Dolci - - _ _ _ _ _ _ Italy

LOANED BY

Hon. Charles A. Schieren.

No. 403 .

ENGLISH LANDSCAPE.

P. Naysmith - - _ _ _ _ London

No. 404 .

SUNDAY AFTERNOON.

H. Von Valkenburgr

Holland

No. 405 .

MOTHER LOTE.

H. Von Valkenburg

Holland 


\section{No. 406. \\ BAVARIAN GIRL.}

Rudolph Epp _ $\quad-\quad$ - $\quad$ - $\quad$ - Munich

No. 407 .

VIEIW OF HAARLEM.

A. Klinkenberg - $\quad$ - $\quad$ - _ _ Holland

No. 408 .

BEGGING MONK.

Max Gaisser - $\quad$ - $\quad$ - $\quad$ - $\quad$ - $\quad$ - $\quad$ Munich

No. 409 .

NYMPHS AND BOATMAN.

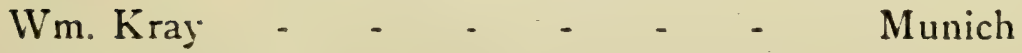

No. 4 ro.

INDUSTRIOUS CHILD.

H. Feshner - - $\quad$ - $_{2} \quad \ldots \quad \ldots$ Munich

No. 4 II.

SUNSET IN WESTPHALEN.

H. Hartung - - - - - - Dusseldorf

No. 4 I 2 .

HUNGARIAN CART.

J. Van der Venner _ _ _ _ Hungary

No. 413 .

PORTRAIT.

Richard Creifelds - _ _ _ _ New York 


\section{LOANED BY \\ John Notman.}

No. 414 .

PORTRAIT-MR. WEDGEWOOD, OF ENGLAND.

Sir Joshua Reynolds - _ _ _ England

No. 415 .

LANDSCAPE IN HOLLAND.

Jacob Maris - _ _ _ _ _ _ Holland

LOANED BY

Mrs. Brett.

No. 416.

THE ANGEL'S IVHISPE.R.

E. Leutze $\quad-\quad \ldots \quad-\quad \ldots \quad-\quad-\quad$ United States

No. 417 .

I WILL; YOU SHAN'T.

Wm. Page - _ _ _ _ _ $\quad$ - United States

LOANED BY

Sidney $W$. Curtis.

No. + IS.

PORTRAIT OF A IADY.

Richard Creifelds - - _ - - New York 
LOANED BY

R. R. Bowker.

\author{
No. 419. \\ STAR AND CRESCENT.
}

F. S. Church - $\quad$ - $\quad$ - $\quad$ - $\quad$ New York

No. 420.

THE WHITE DAY.

Charles Parsons

No. $42 \mathrm{I}$.

THE OLD MILL.

Charles Parsons

New York

LOANED BY

Hon. J. S. T. Stranahan.

No. 422.

PORTRAIT-HON. J. S. T. STRANAHAN.

Daniel Huntington - _ _ _ _ New York

No. 42.3 .

PORTRAIT-IIRS. J. S. T. STRANAHAN.

Daniel Huntington - - _ - $\quad$ New York

No. 424 .

WINTER LANIUSCAPE.

Regis Gignoux - - - _ _ - New York 


\section{LOANED BY \\ Eugene G. Blackford.}

No. 425 :

LAND-LOCKED SALMON.

S. A. Kilbourn - - - - - - Paris

LOANED BY

W. H. Ingersoll.

No. 426 .

RYDAL WATER.

Henry Inman _ _ _ _ _ $\quad$ - United States

LOANED BY

Edward Frossard.

MINIATURES.

; No. 427 .

GEN. GEORCE WASHINGTON.

John Trumbull $\quad$ - $\quad$ - $\quad$ - $\quad$ - United Statés

No. 428 .

GEN. GEORGE WASHINGTON.

John Trumbull - $\quad$ - $\quad$ - United States

No. 429 .

GEORGE AND MARTHA WASHINGTON.

John Trumbull _ _ _ _ - United States

No. +30 .

GEORGE AND MARTHA WASHINGTON.

John Trumbull - $\quad$ - _ United States 
No. 431 .

GFNERAL LAFAYETTE.

John Trumbull - $\quad$ - $\quad$ - United States

No. 432 .

MADAME LEBRUN AND CHILD.

John Trumbull - $\quad$ - $\quad$ - United States

No. 433 .

MRS. GRAHAM, FRENCH LADY.

John Smibert - _ _ _ _ _ U United States

LOANED BY

\section{Richard Dixon.}

No. 434 .

THE SHADOW OF THE CROSS.

J L. Gerome - - - . - - - - Paris

LOANED BY

\section{Sylvester Stearns.}

No. 435 .

THE ADOPTION OF THE CONSTITUTION.

Junius Brutus Stearns - _ _ _ - New York

\section{LOANED BY}

Octavius A. White, M. D.

No. 436 .

GENERAL MARION INVITING THE BRITISH OHFICERS TO DINNER (1826).

John Blake White 
No. 437 .

MRS. MOTTE HANDING THE BURNING ARROWS TO MARION AND LEF TO FIRE HER MANSION

AND SO DISLODGE THE BRITISH (1825).

John' Blake White _ _ _ - South Carolina

No. $43^{8}$.

RESCUE OF AMERICAN PRISONERS NEAR SAVANNAH, GA., BY JASPER AND NEWTON (182t).

John Blake White _ _ _ South Carolina

No. 439 .

"POVERTY COMES IN AT THE DOOR, AND LOYE FLIES OUT AT THE WINDOW."

John Blake White _ _ _ $\quad$ - South Carolina

No. $44^{\circ}$.

VIEW OF BROAD STREET IN CHARLESTON, S. C., IN I830-"Arrival of the Mail."

John Blake White _ _ _ - South Carolina

\author{
LOANED BY \\ Mrs. William S. Gould. \\ No. $4+1$.
}

PORTR.AIT OF BENJAMIN FR.NKLIN.

\title{
LOANED BY \\ C. C. Ruthrauff.
}

No. +42 .

PORTRAIT OF DAVID S. JONES, FIRST CORPORATION COUNSEL, OF NEW YORK CITY.

Thomas Sully

- New York 
No. 443 .

GEORGE WASHINGTON.

Gilbert Stuart

No. 444 .

FEMAIE PORTRAIT.

Thomas Sully

No. 445.

PORTRAIT OF HIS DAUGHTER.

Gerard Terburg _ $\quad$ - $\quad$ - $\quad \ldots \quad$ - Holland

No. 446.

LANDSCAPE AND CATTLE.

L. Tieling - - - _ . - - Holland

No. 447 .

THE FORD AT EVENING.
A. G. Decamps

No. 448.

THE SEINE ON A GRAY DAY.

Fritz Thaulow

- Paris

No. 449 .

NEIV JERSEY AUTUMN.

H. W. Ranger - - - - - New York

No. $45^{\circ}$.

THE PRIZE WINNER.

Charles Wilson Peale - _ _ United States 
LOANED BY

Rev. Charles R. Baker.

No. $45 \mathrm{I}$.

MADONNA.

Giovanni Baptista Salvi, called Il Sassaferrato Italy

LOANED BY

Mrs. Scott and Miss Clements.

No. $45^{2}$.

POR'TRAIT OF THE LATE JOS. ANTHONY, OF PHILADELPHIA.

Gilbert Stuart _ _ _ $\quad$ - $\quad$ - United States

No. 453 .

PORTRAIT OF THE LATE IIRS. JOS. ANTHONY,

Daughter of Michael Hillegas, first Treasurer of the United States.

Gilbert Stuart - $\quad$ - $\quad$ - $\quad$ United States

LOANED BY

Mrs. Phebe A. Hearst.

No. $453 \frac{1}{2}$.

SANTA BARBARA.

Orrin Peck - _ _ _ _ - San Francisco 
72 LOANED BI THOMAS L. ETLLHAL

LOANED BY

Thomas E. Stillman.

No. 454 .

CHARLOTTE CORDAY.

G. Jacquet - - - _ - - - - $\quad$ - Paris

No. 455 .

POLISH SCENE.

J. Pokitonow - $\quad$ - $\quad$ - $\quad$ - $\quad$ - $\quad$ - Paris

No. 456 .

ON THE PINCIO: THE CARDINAL AND THE STUDENTS.

F. Heilbuth - - _ _ _ _ _ - Paris

No: 457 .

PURSUED BY WOLVES.

A. Schreyer : $\quad-\quad \vdots_{-} \quad-\quad-\quad-\quad$ Paris

No. $45^{8}$.

LAWN TENNIS.

F. Heilbuth - - - _ - _ _ Paris

No. 459 .

THE BANDIT'S WIFE.

L. Bonnat - - - _ _ _ - - Paris

No. 460.

EPISODE IN THE FRANCO.GERMAN WAR.

P. Grolleron

- Paris 
No. $46 r$.

VILLE D'AVRAY.

J. B. C. Corot - - - - - - Paris

No. 462 .

FISHER BOY.

Jos. Israels - _ _ _ _ _ _ Holland

No. $4^{6} 3$.

ON THE LOIRE.

C. F. Daubigny - _ _ _ _ _ - Paris

No. $46_{4}$.

FUNERAL ON THE NILE.

E. Fromentin - - - - - - Paris

No. 465 .

SHEPHERD'S RETURN.

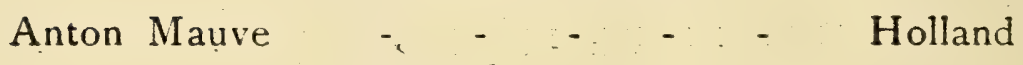

No. 466 .

LOW TIDE.

Jules Dupré -

No. $4^{67}$.

MIDSUMMER.

C. F. Daubigny - $\quad$ - $\quad$ - $\quad$ - $\quad$ - $\quad$ - Paris

No. 468 .

DETAINED BI THE RAIN.

Dagnan Bouveret - $\quad$ - $\quad$ - $\quad$ Paris 


\section{No. 469 .}

THE THREF, COWS.

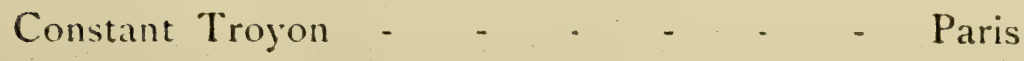

No. 470 .

IN THE FOREST AT FONTAINELLEAU.

N. Diaz - - - - - _ _ - Paris

No. 471 .

VILLE D'AVRAY.

J. B. C. Corot - - - - - - Paris

No. 472 .

THE BETRAYAL. EPISODE, BATTLE OF THE CHUANS.

B. Le Blant - - - _ - - - Paris

No. 473 .

FALLING LEAVES.

Geo. H. Boughton - _ _ _ _ London

No. 474 .

THE WHITE CAP.

Alfred Stevens _ _ _ _ _ _ _ - Paris

No. 475 .

THE FARM.

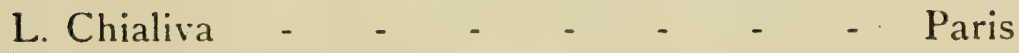

$$
\begin{gathered}
\text { No. } 476 . \\
\text { FORGET ME NOT: }
\end{gathered}
$$

Henry Lerolle 


\section{No. 477 .}

THE OLD TROOPER. IE NEUVIILE'S HORSE. John Lewis Brown _ _ _ _ _ . Paris

No. 478 .

THE DISCUSSION.

David Col - $\quad \ldots \quad \ldots \quad$ - $\quad$ - Antwerp

No. 479 .

WANDERING MUSICIANS.

F. Heilmuth - $\quad$ - $\quad$ - $\quad$ - $\quad$ - $\quad$ - Paris

No. 4 So.

THE CHILDREN'S FRIENDS.

L. Chialiva - _ _ _ _ _ _ - Paris

Nก. $48 \mathrm{r}$.

BURNING BRUSH.

E. Renouf - $\quad-\quad-\quad-\quad-_{-} \quad-\quad-_{-}$Paris

LOANED BY

H. H. Benedict.

No. 482 .

LANDSC.APE IN HOLLAND.

M. Hobbema - _ _ _ _ _ _ _ Holland

No. $4^{8} 3$.

LANDSCAPE WITH WATERFALI.

Jacob Ruysdael _ _ _ _ _ _ _ Holland 


\section{No. $48_{4}$. \\ THE SUNLIT VALE}

A. H. Wyant - - - - - - - New York

No. $4^{8} 5$.

AUTUMN LANDSCAPE, ADIRONDACKS.

A. H. Wyant - - - - - New York

No. 486 .

IN THE DEEP WOODS.

A. H. Wyant - - - - - - - New York

No. 487 .

TIVO COWS IN A POOL.

A. H. Wyant - - - - - - New York

LOANED BY

G. William Rash.

No. 488 .

PORTRAIT OF S. F. B. MORSE.

S. F. B. Morse - - _ _ - United States

LOANED BY

Mrs. Henry C. Polhemus.

No. 489 .

FEMALE HEAD.

Hughes Merle - - - $\quad$ - $\quad$ - $\quad$ - Paris 
No. 490.

CALCULATION.

T. W. Wood - - - - - - New York

No. $49 x$.

GUITAR PLAYER.

S. J. Guy - - - $\quad-\quad-\quad$ - New York

No. $49^{2}$.

NEW CIDER.

T. W. Wood - - - $\quad-\quad$ - $\quad$ - New York

LOANED BY

C. J. Falconer.

No. 493 .

THE TITAN'S GOBLET.

Thomas Cole - _ - _ _ - New York

No. 494 .

SALVATOR ROSA SKETCHING BANDITTI.

Thomas Cole _ _ _ _ _ _ N New York

No. 495 .

PETRARCH'S HOME, ITALY.

J. F. Cropsey _ - - $\quad$ - $\quad$ - $\quad$ - New York

LOANED BY

A. Augustus Low.

No. 496 .

PORTRAIT OF PRESIDENT MADISON.

Gilbert Stuart _ $\quad$ - $\quad$ - $\quad$ - United States 


\section{No. 497 .}

PORTRAIT OF PRESIDENT MONROE.

Gilbert Stuart

No. 49 S.
PORTRAIT OF THE LATE A. A. LOW.

J. Osgood - _ _ _ _ _ - New York

LOANED BY

Henry T. Chapman, Jr.

No. 499.

THE RED MILL.

Claude Lorraine - _ _ _ _ _ Rome

No. 500 .

THE BATTLEFIEI,D.

J. L. T. A. Gericault - _ _ . _ - Paris

No. 501 .

CLASSIC LANDSCAPE-ITALY.

Salvator Rosa

No. 502 .

THE OLD MILL.

Joseph Jefferson - $\quad$ - $\quad$ - $\quad$ Buzzards Bay

No. 503 .

LANDSCAPE.

George Michel - $\quad$ - $\quad$ - $\quad$ - $\quad$ Paris 
No. 504 .

CITY IN HOLLAND.

Abraham Stork _ _ _ _ . . Amsterdam

Nc. 505 .

STADTHOLDERS' COURT-HAGUE.

Abraham Stork and Adrian Van de Velde, Amsterdam

No, 506 .

GYPSY CAMP.

George Morland - $\quad$ - $\quad$ - $\quad \ldots \quad$ - $\quad$ London

No. 507 .

LANDSCAPE AND CATTLE.

Paul Potter - $\quad$ - $\quad$ - $\quad$ - $\quad$ - Holland

No. 508 .

LANDSCAPE AND FIGURES.

George Morland - _ _ _ _ _ _ London

No. 509.

THE COTTAGE DOOR.

George Morland - _ _ _ _ _ London
No. 510.
LADY WITH DOG.

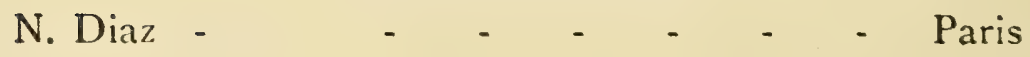

No. 511.

THE LITTLE FIOHER SELLER

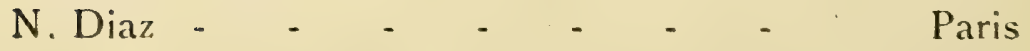


No. 512.

LANDSCAPE WITH FIGURES.

N. Diaz - - - - - $\quad$ - $\quad$ - _ Paris

No. 5 I 3 .

YOUNG WOMAN.

J. B. C. Corot - - - - - - Paris

No. 514.

THE STORM.

G. Courbet - - _ _ _ _ - - Paris

No. 515 .

SCENE IN HOLLAND.

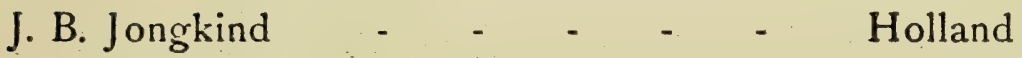

No. 516.

THE VILLAGE PRIEST.

A. G. Decamps - $\quad$ - $\quad$ - $\quad$ - $\quad$ - $\quad$ - Paris

No. 517 :

STREET SCENE.

J. E. C. Roqueplan - - - - - Paris

No. 518 .

THE MORNING GLORY.

William Etty

- - - - - - London

No. 5 I9.

STILI, I.IFE.

A. Vollon - $\quad$ - $\quad$ - $\quad$ - $\quad$ - $\quad$ - Paris 


\section{No. 520 . \\ EVENING. EFFECT.}

Theo. Rousseau _ _ _ _ _ _ _ _ Paris

No. 521 .

FLORA, THE GODDESS OF SUMMER.

Sir Joshua Reynolds - $\quad$ - $\quad$ - $\quad$ - London

No. 522.

THE WANDERER.

Edward Penny _ $\quad$ - $\quad$ - $\quad$ - $\quad$ - London

No. 523 .

VILLA OF M.ECENAS AT TIVOLI.

Richard Wilson

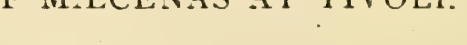

London

No 524 .

THE DUET.

Francis Meiris (The Elder) - - - Holland

No. 525 .

A DUTCH INTERIOR.

Gabriel Metzu _ _ _ _ _ _ _ Holland

No. 526 .

THE WOUNIEEI ST.IC.

Peter Paul Rubens

Holland

No. 527 .

IN THE VINEYARI)

Jan Breughel 
No. 528 .

THE IVRECK.

Claude Joseph Vernet -
No. 529.

A DUTCH LADY.

John Van Ravensteyn - _ _ _ - $\quad$ Holland

No. $53^{\circ}$.

WINTER.

G. Courbet - _ _ _ _ _ _ - $\quad$ - Paris

No. 53 r.

LANDSCAPE.

Gasper Poussin

No. $53^{2}$.

LANDSCAPE.

Homer D. Martin - _ _ _ _ _ New York

No. 533 .

PASTORAL SCENE.

A. P. Ryder - - - - _ - - New York

No. 534 .

SHEEP AND GOATS.

Rosa de Tivoli - $\quad$ - $\quad$ - $\quad$ - $\quad$ - $\quad$ - Italy

No. 535 .

H.RVEST TIME.

Jan Van Goyen _ $\quad \ldots \quad \ldots \quad \ldots$ - $\quad$ - Holland 


\section{No. 536 .}

A BEAUTIFUL MODEL.

Wm. Etty

No. 537 .

AN EARLY LANDSCAPE.

J. B. C. Corot - - - - - - - Paris

No. 538 .

THE STORM.

Salvator Rosa

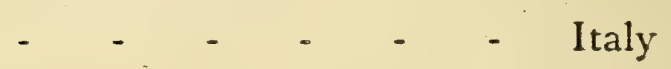

No. 539.

ITALIAN RUINS.

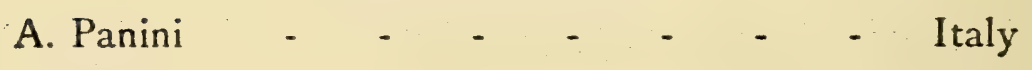

John Wooton - $\quad-\quad \quad-\quad \ldots \quad \ldots \quad$ England

$$
\begin{aligned}
& \text { No. } 54^{\circ} . \\
& \text { ENGLISH HUNTING SCENE. }
\end{aligned}
$$

$$
\text { No. } 5+1 \text {. }
$$

PORTRAIT OF DE WITT CLINTON.

Rembrandt Peale - _ _ _ $\quad$ United States

No. $5+2$.

LANDSCAPE.

H. W. Ranger - - - - - New York

No. $5+3$.

THE GLADE.

Arthur B. Davies - - - - - New York 
No. $5+4$.

ARETHUSA.

Arthur B. Daries - _ - - - New York

No. $5+5$.

CHILDREN OF YESTERYEAR.

Arthur B. Davies - _ - - - New York

No. 546.

PLACE OF THE MOTHERS.

Arthur B. Davies - - _ _ - New York

No. 547 .

THE GODDESS.

Arthur B. Davies - $\quad$ - _ _ _ - New York

No. $5+8$.

LANDSCAPE WITH PIGS.

Arthur B. Davies - $\quad-\quad$ - : New York

No. 549.

SUMMER.

F. B. Williams - - - - - - New York

No. $55^{\circ}$.

NEAR LONDONDERRY, VT.

F. B. Williams

- New York

No. 551 .

RIP VAN WINKLE REACHES THE GNOMES. John Quidor - - - - - - New York 


\section{No. $55^{2}$.}

THE VIGIIANT STLYVESANT, WALI, STREET GATE. John Quidor - _ _ _ _ _ _ N New York

No. 553 .

VOYAGE OF THE GOOD OLOFEE FROM HELL G.TTE TO COMMUNIPAW.

John Quidor - _ _ _ _ _ - New York

No. 554 .

PETER STUYVESANT'S VOYAGE UP THE HUDSON RIVER.

John Quidor - _ - - - - New York

No. 555 .

ICHABOD CRANE PL'RSLED BY THE HEADLESS HORSEMAN OF SLEEPY HOLLOW.

John Quidor - - - - - - - New York

No. 556 :

SCHOOL TIME IN THE ORIENT.

A. G. Decamps - $\quad$ - $\quad$ - $\quad$ - $\quad$ - $\quad$ - Paris

No. 557 .

COURTSHIP OF MILFS STANDISH.

No. 55 S.

GEN. WAYNE REFLSING THE LAST BLANKET,

O. A. Bullard _ _ _ _ _ New Jork

No. 559.

LANISC.IPE IN THE CATSKILLS.

Henry Inman

New Jork 
No. 560 .

PORTKAIT (IF GILBERT STLART AT EIGHTEEN YEARS OF AGE.

Gilbert Stuart _ _ _ _ _ _ United States

No. $5^{6} \mathrm{I}$.

PORTRAIT OF ROBERT FUITON.

Robert Fulton

- New York

No. $5^{62}$.

ISAAC DEPEYSTER.

F. V. Doornik _ _ _ _ _ $\quad$ United States

No. $5^{6} 3$.

MARTHA DANIRIDGE, EIGHT AND ONE.HALF YEARS OL.D, afterwards Martha Washington.

John Smybert, 174I _ _ _ - United States

No. $5^{6} 4$.

SAMUEL ADAMS.

J. S., 1764 - $\quad-\quad+\quad-\quad$ - United States

No. 56.5 .

ON THE RHINE.

E. IV. Nichols - - - - - New York

No. 560 .

ANNIE FRANCIS, Mother of Col. Tench Tilghman.

No. $5^{67}$.

IN HIS GIORY.

A. Blythe - - - - _ - New York 
No. $5^{638}$

PORTRAIT OF A GENTLEMAN.

A. Powell, $1843, \ldots \ldots \ldots$ - $\quad \ldots$ New York

No. $5^{69}$.

PORTRAIT OF CHAS. CLINTON.

Matthew Pratt, 1769, - - - - New York

No. 570 .

PORTRAIT OF MRS. CHAS. CLINTON.

Matthew Pratt, г $; 69$, - - _ - New York

No. $57 \mathrm{I}$.

ANN N. DE PEYSTER, SISTER OF ISAAC DE PEYSTER.

F. V. Doornik, _ $\quad-\quad-\quad-\quad \ldots \quad \ldots \quad$ New York

No. 572 .

THOMAS ABTHORPE COOPER.

John Wesley Jarvis, - - - _ - - New York

No. 573 .

MRS. THOMAS ABTHORPE COOPER.

Wm. Dunlap, - - - - - - New York

PRESENTED BI

Abraham Abraham.

No. 57 .

THE SHEPHERIESS.

Ridgeway Knight. - _ _ _ _ New lork 


\section{PROPERTY OF \\ Brooklyn Institute of Arts and Sciences}

No. 575 .

BROOKLYN IN ISIG.

Guy, - - - - - - - - - New York

\section{LOANED BY \\ Heinigke \& Bowen,}

No. 576 .

A Mosalc WINDOW, "THE RETURN OF THE DOVE."

MADE BY

HEINIGKE \& BOWEN.

\section{Tiffany. Glass \& Decorating Co.}

No. 577 .

A COLORED GLASS IVINDOW.

The Edward S. Steele memorial for the Philadelphia High School, executed in the studios of the Tiffany Glass and Decorating Company of New York.

No. 578 .

A CASE OF TIFFANY FAVRILE GLASS.

Twenty-four objects.

$$
\begin{aligned}
& \text { No. } 579 . \\
& \text { SUPERIMPOSED MOTIVES. }
\end{aligned}
$$

These pieces are obtained by combining layers of glass differently charged with metals, in some cascs so hexvily charged that the glass has taken on the appearance of met:ll rather than that of glass.

$$
\text { No. } 5^{80} \text {. }
$$

FLOWER MOTIVES.

The ornamentation, although produced by the means of oparpe glass, does not interfere with the transparency of the pieces, hecause it is composed of threads of exceeding fincness, and so rlistributed that thert is a line of clear glass between each thread. 


\section{No. $5^{81}$. \\ PEACOCK MOTIVES.}

This glass is made of five different varieties, namely: two of avanturine, two of transparent, and one of opaque.

The decoration is produced while the pieces are in a molten state, by inlaying them with threads, and manipulating this threading to a given design. When the blowing is finished, the pieces are then made iridescent by exposing them to the fumes of metals.

No. $5^{82}$.

\section{COLOR MOTIVES.}

The variation in color in these pieces is due not to a different mixture of glasses, but to a difference in the degree of heat to which the one mixture has been subjected.

\section{No. $5^{8} 3$.}

\section{A CASE OF TIFFANY FAVRILE GLASS.}

Six lamps, three plaques, four vases. A model of the Mfosaic decoration in the rotunda of the Chicago Public Library. A figure panel in glass mosaic. Seven examples of glass mosaic. Four cartoons for colored windows-The Elements-earth, air, fire and water.

All the above objects are from the studios of the Tiffany Glass and Decorating Company, and were made under the personal supervision o Mr. Louis C. Tiffany. 



$\sum$
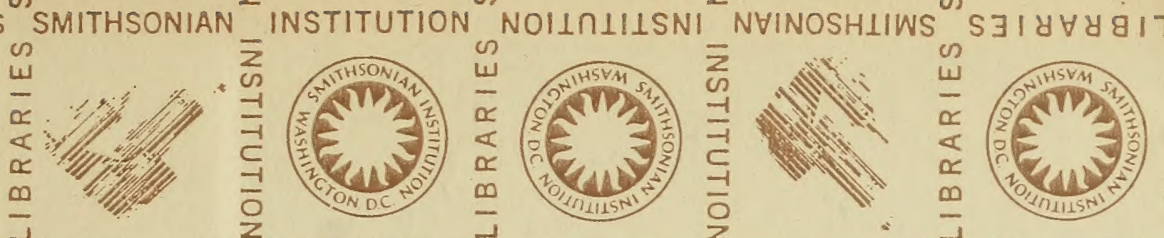

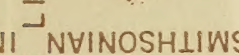

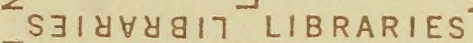

SMITHSONIAN
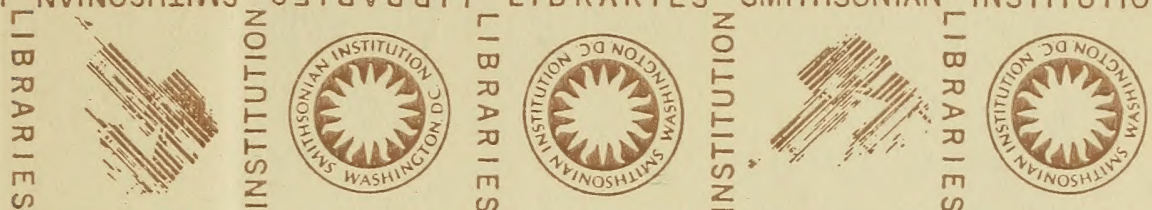

$z$
0
5
5
$E$
E
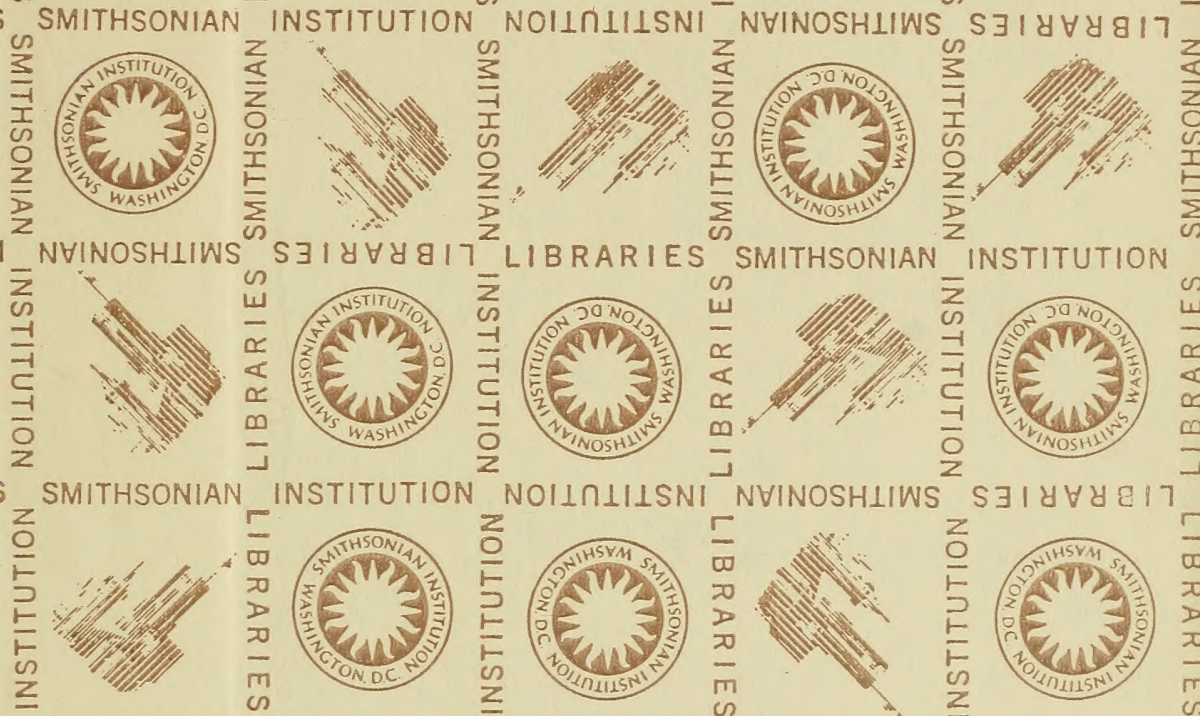

NI
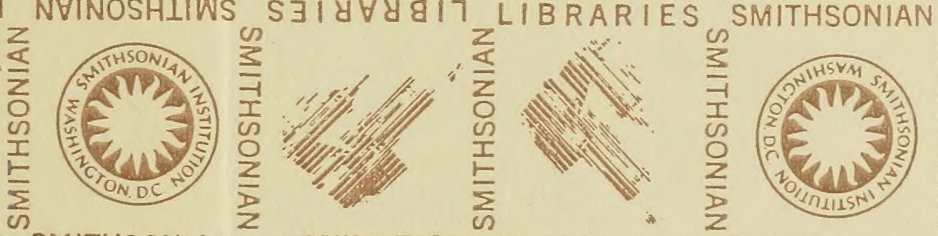

$\frac{1}{\frac{1}{2}}$

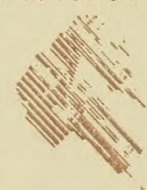

$\infty$
3
7
1
0
0
2
2
2
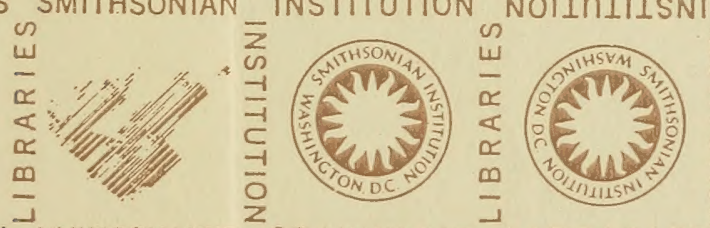

NHINOSHIIWS
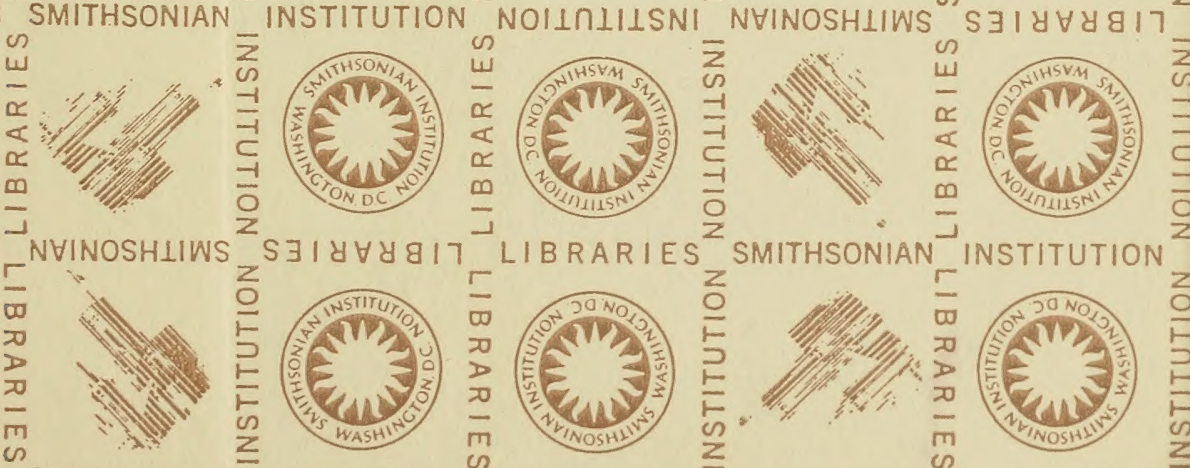

$\frac{-1}{0}$

SMITHSONIAN

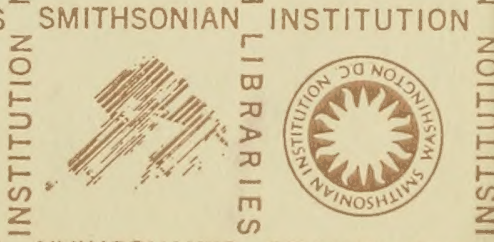

$S$ SMITHSONIAN INSTITUTION NOIINLILSNI 
SMITHSONIAN INSTITUTION LIBRARIES

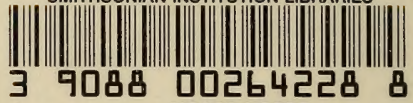

nmaa N5020.N5B87

Illustrated catalogue of paintings and o 